\title{
Studying Learning at Scale with the ASSISTments TestBed
}

\section{Korinn S. Ostrow}

Worcester Polytechnic Institute

Worcester, MA 01609, USA

ksostrow@wpi.edu

\section{Abstract}

An interactive demonstration on how to design and implement randomized controlled experiments at scale within the ASSISTments TestBed, a new collaborative for educational research funded by the National Science Foundation (NSF). The Assessment of Learning infrastructure (ALI), a unique data retrieval and analysis tool, is also demonstrated.

\section{Author Keywords}

ASSISTments TestBed, Randomized Controlled

Experimentation at Scale, Authentic Learning

Environments, Assessment of Learning Infrastructure.

Permission to make digital or hard copies of part or all of this work for personal or classroom use is granted without fee provided that copies are not made or distributed for profit or commercial advantage and that copies bear this notice and the full citation on the first page. Copyrights for third-party components of this work must be honored. For all other uses, contact the Owner/Author.

Copyright is held by the owner/author(s)

L@S 2016, April 25-26, 2016, Edinburgh, Scotland UK

ACM 978-1-4503-3726-7/16/04

http://dx.doi.org/10.1145/2876034.2893404

\section{ACM Classification Keywords}

K.3.1 Computer Uses in Education; H.5.2 User Interfaces - Training, help, and documentation.

\section{Introduction}

This demonstration serves as an interactive tutorial highlighting the ease with which researchers can conduct educational experiments at scale using the ASSISTments TestBed [3]. The TestBed is a unique collaborative funded by an NSF SI2 grant [1] that allows researchers to design controlled experiments with student level randomization and to implement them at scale by leveraging ASSISTments' pre-existing content and user population. ASSISTments, a popular online learning platform, has over 50,000 student users per year and offers an authentic learning environment in which researchers can observe the effects of learning interventions within online learning [2]. This demonstration will also offer a hands-on trial of the Assessment of Learning Infrastructure (ALI), a new data retrieval and analysis tool to be introduced at the Learning Analytics \& Knowledge Conference [4].

\section{Intellectual Merit of the TestBed}

Prior to recent advances in learning technologies, conducting educational research at scale was tumultuous. Research carried high costs, was restricted by teacher-level randomization, and was often obtrustive to natural learning processes. However, 


\section{Features of the ASSISTments TestBed}

Funded: The TestBed is funded by an NSF SI2 Grant [1], meaning low (or no) out of pocket costs for researchers.

IRB: ASSISTments' IRB allows for research designs qualifying as 'normal instructional practice.' Researchers simply need approval from their IRB to access anonymized data.

Simple UI: ASSISTments offers content authoring tools that are easy to use and do not require complex programming knowledge [2].

\section{Collaborative Laboratory:}

The TestBed brings researchers together with students and teachers to study effective learning interventions while iteratively improving ASSISTments content and the user experience.

Data Retrieval: The Assessment of Learning Infrastructure (ALI), a state of the art data retrieval and analysis tool, streamlines data cleaning and analysis [4]. online learning platforms like ASSISTments offer authentic learning environments in which students receive immediate feedback on classwork or homework while teachers enhance their lessons through robust data driven reports. Built around the benefits inherent to the ASSISTments platform, the new ASSISTments TestBed [3] revolutionizes educational research by making it simple for researchers to design and implement low cost, high power, randomized controlled experiments that are unobtrusive to student learning.

\section{Demonstration Details}

Researchers from around the world already successfully use the ASSISTments TestBed to study educational interventions at scale. Through this demonstration, we hope to expand the benefits of this infrastructure to a broader research community. This demonstration will cover the following points in an interactive fashion:

- An overview of ASSISTments and its importance to the TestBed.

- Highlights from studies currently running in the TestBed, outlining the 5 -stage research process (Figure 1).

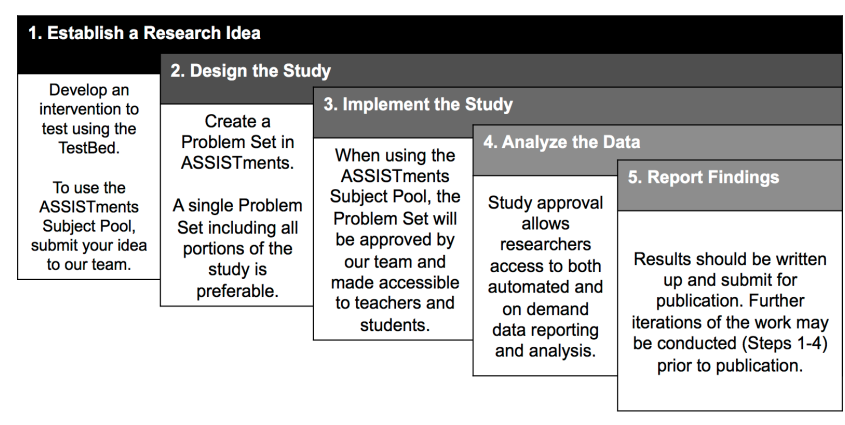

Figure 1: The five stages of conducting research within the ASSISTments TestBed
- Introduction to accessing ASSISTments' subject pool and implementing a study.

- Hands-on session building and altering content within ASSISTments.

- Hands-on design and implementation of a simple study, with demonstration participants as subjects.

- Hands-on introduction to ALI, a unique tool for automated data retrieval and analysis [4].

Researchers will leave this demonstration with an understanding of how to use the ASSISTments TestBed to study learning at scale.

\section{Acknowledgements}

We acknowledge funding from the NSF (1440753, $1252297,1109483,1316736,1535428$, \& 1031398), the U.S. Dept. of Ed. (R305A120125, R305C100024, \& P200A120238), and ONR's "STEM Grand Challenges."

\section{References}

1. Heffernan, N. (2014). SI2-SSE: Adding Research Accounts to the ASSISTments Platform: Helping Researchers Do Randomized Controlled Studies with Thousands of Students. (NSF \#1440753). http://goo.gl/lv2Ndi

2. Heffernan, N. \& Heffernan, C. (2014). The ASSISTments Ecosystem: Building a Platform that Brings Scientists and Teachers Together for Minimally Invasive Research on Human Learning and Teaching. Int J of AIED. 24(4), pp. 470-497.

3. Ostrow, K. \& Heffernan, C. (2015). ASSISTments TestBed Resource Guide. https://goo.gl/9GHuiS

4. Ostrow, K., Selent, D., Wang, Y., Van Inwegen, E., Heffernan, N., \& Williams, J.J. (In Press). The Assessment of Learning Infrastructure (ALI): The Theory, Practice, and Scalability of Automated Assessment. To be included in the Proceedings of the 6th Int Conf on LAK. 